\title{
Modeling the bremsstrahlung of 30-60 MeV electrons. Source term calculation
}

\author{
M. MIREA ${ }^{1,2}$, O. BAJEAT $^{1}$, F. CLAPIER $^{1}$, M. HASSAINE $^{2,3}$, F. IBRAHIM $^{1}$, \\ A.C. MUELLER ${ }^{1}$, N. PAUWELS ${ }^{1,4,{ }^{*}}$, J. PROUST ${ }^{1}$, D. VERNEY ${ }^{1,5}$, R. ANTONI ${ }^{6}$, \\ L. BOURGOIS ${ }^{6}$, S. KANDRI-RODY ${ }^{1,7}$
}

(Manuscript received 18 June, accepted 17 October 2002)

\begin{abstract}
The photofission process has been recently considered for the production of neutron rich isotopes and the development of radioactive beams. The radioprotection hazard should be studied accordingly. A survey of the radiative electron energy loss theory is reported in order to estimate numerically the bremsstrahlung production of thick targets. The resulted bremsstrahlung angular and energy theoretical distributions delivered from $W$ and $U C x$ thick converters are presented and compared with previous results. This study is focused on initial kinetic energies of the electron beam included in the range $30-60 \mathrm{MeV}$, suitable for the production of large photon yields able to induce the ${ }^{238} \mathrm{U}$ fission. The source term for $50 \mathrm{MeV}$ incident electrons is reported for radioprotection purposes.
\end{abstract}

RÉSUMÉ Modélisation du rayonnement de freinage pour des électrons de 30 à $60 \mathrm{MeV}$. Calcul du terme source

La photofíssion est un processus à l'étude actuellement pour des applications dans les domaines de la production d'isotopes riches en neutrons et des faisceaux d'ions radioactifs. La radioprotection associée fait également l'objet d'une étude. Un compte rendu de la théorie concernant la perte d'énergie des électrons est reporté en vue d'estimer numériquement le rayonnement de freinage émis dans des cibles épaisses. Les distributions angulaires et en énergies du rayonnement de freinage théorique sont présentées et comparées avec des résultats antérieurs. Cette étude est focalisée sur des faisceaux d'électrons d'énergies comprises entre 30 et $60 \mathrm{MeV}$, qui permettent de grandes productions de photons capables d'induire la fission de ${ }^{238} U$. Le terme source pour $50 \mathrm{MeV}$ électrons incidents est reporté en vue d'applications de radioprotection.

\footnotetext{
* Corresponding author.

1 Institut de Physique Nucléaire, 91406 Orsay Cedex, France.

2 Institute of Physics and Nuclear Engineering. P.O. Box MG-6, Bucarest, Romania.

3 Universilé de Tours, 37200 Tours, France.

4 Laboratoire pour l'Utilisation du Rayonnement Électromagnétique, 91898 Orsay Cedex, France.

5 Grand Accelérateur National d'lons Lourds, 1402। Caen Ccdex. France.

6 Commissariat à l'Énergie Atomique Saclay, 91191 Gif-sur-Yvette, France.

7 Université Chouaib Doukkali, B.P. 20, El Jadida, Maroc.
} 


\section{Introduction}

Nuclear fission of heavy nuclei is a process that allows the production of neutron rich isotopes. Exploiting this property, a source of nuclei near the stability limit can be conceived. The feasibility study of an exotic nuclei source involving the fission process represents the main objective of the PARRNe (Production d'Atomes Radioactifs Riches en Neutrons) R\&D project. Two different concepts have been studied to build such a source. The first one is based on the neutron-induced fission of ${ }^{238} \mathrm{U}$. A high neutron flux can be produced by using the deuteron break-up reaction. Experimental and theoretical results concerning the analysis of this concept are reported by Clapier et al. (1998), Kandri-Rody et al. (2000), Mirea et al. (2001). Secondly, in a competitive manner, it is intended to study the feasibility of an exotic nuclei source by involving the bremsstrahlung-induced fission following the conceptual mechanism proposed by Diamond (1999). In a first step, an electron beam of $30-60 \mathrm{MeV}$ is focussed onto a $\mathrm{W}$ converter or onto the uranium carbide target (UCx) itself to deliver a bremsstrahlung radiation. In the second step, the photons induce the fission of the ${ }^{238} \mathrm{U}$. To realize such an experimental combination, accurate knowledge of the bremsstrahlung spectrum is essential. After a convincing test performed with one of the CERN LINACS, a $50 \mathrm{MeV}$ and $10 \mu \mathrm{A}$ electron LINAC is projected at Orsay in order to generate photon induced fission at the PARRNe facility. This work is mainly concerned on the bremsstrahlung distributions evaluation in order to serve several radioprotection purposes: radiation source term, radioactive levels, estimating the electron conversion and estimating the radioactive nuclei yields in the target. Since the experimental systematic of bremsstrahlung spectra is not accurate, and the coverage is too sparse, one must rely on the theoretical results. The next section is devoted to this subject.

\section{Theoretical guideline}

In this section, the main steps used to deduce the bremsstrahlung emission from targets are overviewed. The distributions of the electrons inside a target are obtained using single and multiple scattering theories. The differential bremsstrahlung emission is determined by selecting formulas and correction factors that give the best estimate for the cross-section. The angular distribution of the photons is finally deduced by applying the folding theorem between the angular electron spread and the differential bremsstrahlung cross-section. The slowing down of the electrons is estimated to obtain the mean energy along the range. The electron spread is taken into account by mean of the Landau's distribution. Integrating the irradiated flux along the electron range, 
the absorption of the continuous bremsstrahlung in the converter (or in the target) is taken into account. The shape of the converter is considered as cylindrical. Intensities of second-generation photon spectra are produced up to $10 \%$ of the total field with small energies which are not soft enough to induce fission (Tsai and Whitis, 1966), so, this effect is disregarded. In the following only a survey of the main steps of the theories used to determine the bremsstrahlung productions is presented, an extensive overview being reported by Mirea et al. (2002).

The angular and the projected distributions of the electrons at a depth $d$ inside the target are obtained following the prescriptions of Knop and Paul (1968) concerning the validity of the Mott's cross-section and the Molière's distributions. Therefore, if the depth where the electron distribution is determined is very small, that means the condition $d<<1 /(\sigma N)$ is fulfilled (where $\sigma$ is the cross-section and $N$ the number of atoms per volume unity), we use the Mott cross-section:

$$
\frac{\mathrm{d} \sigma}{\mathrm{d} \Omega}=\frac{\mathrm{Z}^{2} r_{0}^{2}}{4 \sin ^{4} \frac{\theta}{2}} \frac{1}{E^{2}\left(1-\frac{1}{E^{2}}\right)^{2}} R(E, Z, \theta) r(E, Z, \theta)
$$

where $R(E, Z, \theta)$ is the dimensionless ratio between the cross-section at high energy and the Rutherford's classical Coulomb scattering cross-section, $r(E, Z, \theta)$ denotes a correction due to the shielding, $r_{0}=2.8 \times 10^{-13} \mathrm{~cm}$ is the classical electron radius and $E$ is the total energy of the electron in $m c^{2}$ units. The ratio $R(E, Z, \theta)$ is calculated with the model of McKinley and Feshbach (1948) while dimensionless values for $r(E, Z, \theta)$ are extracted from the literature (Scott, 1963). This formula gives the differential cross-section in $\mathrm{cm}^{2} / \mathrm{sr}$ units. Throughout this paper, $Z$ and $A$ denote the atomic and the mass numbers of the converter. Using this formula, the probability that the electron is not deviated up to the depth $d$ was also obtained and the projected distribution (to compute the lateral deflection) was deduced. On the other hand, if the depth $d$ becomes greater or equal to $1 /(\sigma N), N$ being the number of scattering atoms per $\mathrm{cm}^{3}$, we used the multiple scattering theory of Molière (Scott, 1963).

An interpolation is realized in the plural scattering domain between the expressions deduced for single scattering and multiple scattering.

The choice of the bremsstrahlung differential cross-section in photon energy and angle formulas as a function of the electron energy was guided by the 
prescriptions given by Koch and Motz (1950) and Berger and Seltzer (1970):

$$
\frac{\mathrm{d}^{2} \sigma}{\mathrm{d} k \mathrm{~d} \Omega}= \begin{cases}\mathrm{A} f_{E} \frac{\mathrm{d}^{2} \sigma^{2 \mathrm{BN}}}{\mathrm{d} k \mathrm{~d} \Omega} & \text { for } E_{e}<2 \mathrm{MeV} \\ \mathrm{A} \frac{\mathrm{d}^{2} \sigma^{2 \mathrm{BN}}}{\mathrm{d} k \mathrm{~d} \Omega} & \text { for } 2 \mathrm{MeV}<E_{e}<50 \mathrm{MeV} \text { and } \gamma \geq 15 \\ \mathrm{~A} \frac{\mathrm{d}^{2} \sigma^{2 \mathrm{BS}}}{\mathrm{d} k \mathrm{~d} \Omega} & \text { for } 2 \mathrm{MeV}<E_{e}<50 \mathrm{MeV} \text { and } \gamma<15 \\ \frac{\mathrm{d}^{2} \sigma^{2 \mathrm{BN}}}{\mathrm{d} k \mathrm{~d} \Omega} & \text { for } 50 \mathrm{MeV}<E_{e}<100 \mathrm{MeV} \text { and } \gamma \geq 15 \\ \frac{\mathrm{d}^{2} \sigma^{2 \mathrm{CS}}}{\mathrm{d} k \mathrm{~d} \Omega} & \text { for } 50 \mathrm{MeV}<E_{e}<100 \mathrm{MeV} \text { and } \gamma<15\end{cases}
$$

where specific forms of the cross-sections are available in the cited work (Koch and Motz, 1950). In the previous relation, $\sigma$ is the cross-section, $k$ is the energy of the emitted bremsstrahlung ray (which is measured in $m c^{2}$ units in the original paper), $E_{e}$ is the initial kinetic energy of the electron and $\gamma$ is a dimensionless screening factor which depends on the atomic number of the target nucleus, the initial and final energies of the electron during the interaction. The dimensionless Elwert factor $f_{E}$ is a correction for non-relativistic energies.

The folding theorem is applied to obtain the angular distribution. As prescribed by Scott (1963), the folding theorem for spatial distributions reads:

$$
\begin{aligned}
F(\theta, \varphi)= & F\left(\arctan \sqrt{\tan ^{2} \psi_{x}+\tan ^{2} \psi_{y}}, \arctan \frac{\tan \psi_{y}}{\tan \psi_{x}}\right) \\
= & \int_{-\pi}^{\pi} \mathrm{d} \psi_{1 x} \int_{-\pi}^{\pi} \mathrm{d} \psi_{1 y} F_{1}\left(\arctan \sqrt{\tan ^{2} \psi_{1 x}+\tan ^{2} \psi_{1 y}}, \arctan \frac{\tan \psi_{1 y}}{\tan \psi_{1 x}}\right) \\
& \times F_{2}\left[\arctan \sqrt{\tan ^{2}\left(\psi_{x}-\psi_{1 x}\right)+\tan ^{2}\left(\psi_{y}-\psi_{1 y}\right)}, \arctan \frac{\tan \left(\psi_{y}-\psi_{1 y}\right)}{\tan \left(\psi_{x}-\psi_{1 x}\right)}\right]
\end{aligned}
$$

where

$$
\begin{aligned}
& \tan \psi_{x}=\tan \theta \cos \varphi \\
& \tan \psi_{y}=\tan \theta \cos \varphi
\end{aligned}
$$

and $F_{1}$ and $F_{2}$ refer to angular spatial distributions, while $F$ is the folded distribution. 
Due to the axial symmetry of the bremsstrahlung emission and the angular electron distributions, the folding integral can be reduced (overlooking the dummy $\varphi$-coordinate) to

$$
\begin{aligned}
\frac{\mathrm{d}^{2} \sigma_{f}(\theta)}{\mathrm{d} k \mathrm{~d} \Omega}= & \int_{-\pi}^{\pi} \mathrm{d} \psi_{1 x} \int_{-\pi}^{\pi} \mathrm{d} \psi_{1 y} \frac{\mathrm{d}^{2} \sigma\left[\arctan \sqrt{\tan ^{2}\left(\psi_{x}-\psi_{1 x}\right)+\tan ^{2}\left(\psi_{y}-\psi_{1 y}\right)}\right]}{\mathrm{d} k \mathrm{~d} \Omega} \\
& \times F\left(\arctan \sqrt{\tan ^{2} \psi_{1 x}+\tan ^{2} \psi_{1 y}}\right)+P(\theta=0, d) \frac{\mathrm{d}^{2} \sigma(\theta)}{\mathrm{d} k \mathrm{~d} \Omega}
\end{aligned}
$$

where we chose that $\psi_{x}=\psi_{y}=\arctan (\tan \theta / \sqrt{2})$ in order to obtain a symmetric double folding integral, which is more suitable for a numerical treatment. Here, $P(\theta=0, d)$ denotes the probability that the electron is not deviated at the depth $d$ in the target, $F$ (depending only upon the cylindrical coordinate $\theta$ ) is the angular distribution of the electron. Due to the strong dependence of the final cross-section on the electron angular distribution, this integral is effected in 200 Gauss Legendre mesh point's quadratures.

The mean energy of the electron along the path can be obtained using slowing down theories. Mainly two effects produce the slowing down of the electron: the inelastic collision and the bremsstrahlung production.

The mean energy loss due to inelastic collisions is given by the following numerical formulas appropriate for low and high incident energies (Knop and Paul, 1968). The Bohr expression for the mean energy loss reads

$$
-\frac{\mathrm{d} \bar{E}_{c}}{\mathrm{~d} x}=0.306 \rho\left(\frac{Z}{A}\right) \beta^{-2} \ln \left(1.16 \frac{E_{k}}{I}\right)
$$

for $\beta<0.5$. The available Bethe-Bloch expression in the relativistic region is

$$
\begin{aligned}
-\frac{\mathrm{d} \bar{E}_{c}}{\mathrm{~d} x}= & 0.153 \rho \frac{Z}{A} \beta^{-2}\left[\ln \frac{E_{k}\left(E_{k}+m \mathrm{c}^{2}\right)^{2} \beta^{2}}{2 I^{2} m \mathrm{c}^{2}}+\left(1-\beta^{2}\right)\right. \\
& \left.-2 \sqrt{1-\beta^{2}}-1+\beta^{2} \frac{1}{8}\left(1-\sqrt{1-\beta^{2}}\right)^{2}-\delta\right]
\end{aligned}
$$

for $\beta \geq 0.5$. The mean energy loss is given in $\mathrm{MeV} / \mathrm{cm}$, the density $\rho$ is considered in $\mathrm{g} / \mathrm{cm}^{3}$, here $E_{k}$ is the electron kinetic energy in $\mathrm{MeV}, m c^{2}=0.51 \mathrm{MeV}$, and $I$ (MeV units) are the ionization potentials which are listed in the literature (Ahlen, 1980). The parameter dimensionless $\delta$ is known as a density effect. The semiempirical form of $\delta$ originally proposed by Sternheimer (1952) is still used (Ahlen, 1980 ). The parameter $\beta$ is the velocity of the electron divided by the velocity of the light. 
For the radiative emission, relativistic and classical theories were used to determine the slowing down. An extensive list of the detailed formulas used in the present simulations are displayed in the work of Pages et al. (1972).

An estimation of the electron beam straggling must be realized because in traversing a given thickness of the scattering medium, the electrons undergo discrete loss. This straggling distribution can be conveniently parameterized with the Landau's distribution (Tabata and Ito, 1979). Consequently, the differential cross-section for the emission of a photon quantum was folded with the electron energy distributions at a given depth.

As mentioned by Knop and Paul (1968), for thick foils theoretical calculations are very difficult and only for complete diffusion it is possible to give an analytical expression for the angular distribution

$$
F(\theta)=\frac{3}{4 \pi}(0.717+\cos \theta) \cos ^{2} \theta
$$

In order to improve the results at the end of the range, the angular distribution is varied linearly from that obtained with the multiple scattering formula to that available for complete diffusion. This linear variation begins when the electron average energy equals $1 / 4$ of the initial beam energy in order to reach the complete diffusion at the end of the electron range.

The endpoint region (high frequency) of the bremsstrahlung spectrum is corrected using the Fano formula (Koch and Motz, 1950):

$$
\frac{\mathrm{d}^{2} \sigma}{\mathrm{d} k \mathrm{~d} \Omega}=\frac{2 Z^{3} r_{0}^{2} \beta \sin ^{2} \theta^{1+\frac{1}{2} E(E-1)(E-2)(1-\beta \cos \theta)}}{137^{2} k^{3} E^{3}} \frac{(1-\beta \cos \theta)^{4}}{(1-2)}
$$

A linear interpolation is used in the way similar to that used by Berger and Seltzer (1970) in the region $0.85 E_{e}<k<E_{e}, E_{e}$ being the kinetic energy of the electron. The different cross-section formulas are smoothly joined during the passage of their appropriate limits of validity by mean of a linear interpolation. This procedure avoids abrupt variations of the cross-section in the vicinity of the limits of validity. $E$ is the total incident electron energy. Both $k$ and $E$ are given in $m c^{2}$ units.

The effect of mixtures can be included in computing the lateral and angular deflections by replacing $N Z^{2}$ by $\sum_{i} N_{i} Z_{i}^{2}$ summed on different atomic species (Scott, 1963), $N_{i}$ being the number of atom of the specie $i$ in the volume unity. To compute the slowing down and the bremsstrahlung cross-section, the effective values of the target parameters can be deduced using the Bragg rule (Leo, 1987; 
Sternheimer, 1961) which stipulates that the stopping of the individual elements are approximately additive. The same rule is valid for the gamma absorption coefficients (Davisson, 1968).

\section{Results and discussion}

The primary purpose of our calculations was to obtain the bremsstrahlung spectrum of interest for the design of a neutron rich nuclei source based on the gamma induced fission of ${ }^{238} \mathrm{U}$. For that purpose, high gamma yields at energies around $15 \mathrm{MeV}$ are necessary, focussed as best as possible in the forward direction, in order to produce a large number of fission events in the fissionable target placed in the vicinity of the converter. A new numerical tool was conceived to estimate quickly these spectra. Using the mentioned theories, the electron energy spread together with the angular distributions are determined in 50 depth values in the target material. It can be mentioned that concerning the slowing down process, our numerical results agree within few percents with the previous published values (Pages et al., 1972). For each depth value, the energy and angular distributions of the emitted bremsstrahlung rays are calculated in a large array of photons energies ( 25 values) and $\theta$-coordinates ( 25 values). The final differential cross-section of the bremsstralung emission are obtained by integrating on the electron energy spread using spline interpolations between the grid of values determined previously. For each photon energy and $\theta$-angle, the number of bremsstrahlung quanta is obtained by integrating the interpolated values obtained for the final differential cross-section. Briefly, to obtain the bremsstrahlung distribution from converters, the double differential cross-section is integrated along the electron path taking into consideration the electron energy spread, the slowing down and the gamma attenuation in the converter material. As for the radiation field, the bremsstrahlung spectrum emitted at $0^{\circ}$ is harder and more intense that the spectrum obtained in other directions, this evaluation will help to conceive the radiative shielding of the source in the critical conditions required by the electron LINAC being built at Orsay Tandem. In the following work, the bremsstrahlung emissions of cylindrical $W$ and UCx targets are simulated for initial energies of the electrons included in the interval $30-60 \mathrm{MeV}$. The converter thicknesses are considered twice the range of the electron to allow comparisons with previous published results. In Figure 1 , the spectra $\mathrm{d}^{2} N / \mathrm{d} \Omega \mathrm{d} E_{\gamma}$ of the bremsstrahlung emerging at various directions from a thick $W$ target for incident electron energy of $60 \mathrm{MeV}$ are represented. Here $N$ is the number of photons, $E_{\gamma}$ is the gamma energy in $\mathrm{MeV}$. At the small angles $0^{\circ}$ and $5^{\circ}$, Monte-Carlo simulations (Berger and Seltzer, 1970) for $\mathrm{W}$ target of same thickness can be compared. As expected, the high-energy bremsstrahlung yields are larger in the vicinity of a small region around the $0^{\circ}$ emergence angle. Only electrons that have lost little 


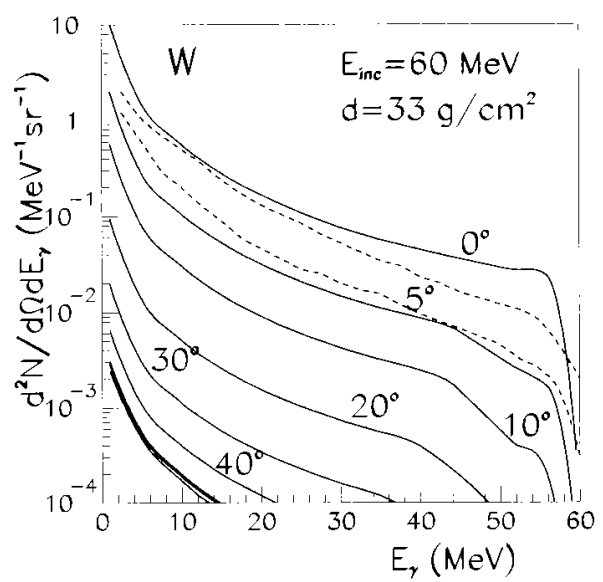

Figure 1 - Angular and energy distributions of the bremsstrahlung emission per $60 \mathrm{MeV}$ incident electron. The cylindrical converter is made from $W$, the thickness is $33 \mathrm{~g} / \mathrm{cm}^{2}$. The diameter is equal to the thickness. The full lines correspond to the present calculations. Some emission angles are marked on the plot. The results corresponding to $50^{\circ}$ up $80^{\circ}$, in steps of $10^{\circ}$, are also displayed but the yields are lower. The dotted lines are extracted from the work of Berger and Seltzer (1970) for (0-0.5) and (0-5).

Distributions angulaires et en énergie du rayonnement de freinage par électron incident de $60 \mathrm{MeV}$. Le convertisseur cylindrique est formé de W, l'épaisseur est de $33 \mathrm{~g} / \mathrm{cm}^{2}$. Le diamètre est égal à l'épaisseur. Les lignes pleines correspondent au calcul actuel. Quelques valeurs des angles d'émission sont marquées sur la figure. Les résultats qui correspondent à l'intervalle de $50^{\circ}$ jusqu'à $80^{\circ}$, en pas de $10^{\circ}$, sont aussi dessinés, mais les rendements sont plus petits. Les lignes discontinues sont extraites du travail de Berger et Seltzer (1970) pour $(0-0,5)^{\circ}$ et $(0-5)^{a}$.

energy and, consequently, have not yet deflected much by multiple scattering can emit the high-energy bremsstrahlung. Generally, our forward direction values of the spectra agree with those of Berger and Seltzer (1970). However, larger deviations appear in the high-energy region, where $E_{\gamma}>0.9 E_{i n c}, E_{i n c}$ being the energy of the beam. These differences can be understood as follows: in the highenergy region, we used different methods to correct the cross-section. In this paper, the analytical Fano formula is kept while in the cited work (Berger and Seltzer, 1970), an interpolation with experimental data was done. In Figure 2, the same quantities are plotted for incident electron energy of $30 \mathrm{MeV}$. Similar trends as in Figure 1 are displayed, but the yield at $15 \mathrm{MeV}$ is lowered by a factor 3 . From these examples, we illustrated the degree of accuracy of our simulations. The present study being mainly an exploratory analysis of the neutron rich nuclei source, results with accuracy better than $30 \%$ are convenient in preparing the experimental work. Our interest is now focussed on initial energies of $50 \mathrm{MeV}$, because from considerations which are not of physical nature, it is expected that our future 


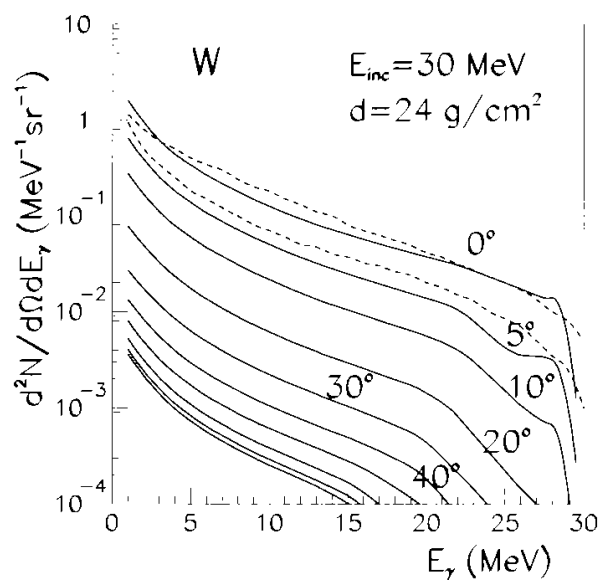

Figure 2 - Angular and energy distributions of the bremsstrahlung emission per 30 MeV incident electron. The cylindrical converter is made from $W$, the thickness is $24 \mathrm{~g} / \mathrm{cm}^{2}$. The diameter is equal to the thickness. The full lines correspond to the present calculations. Some emission angles are marked on the plot. The results corresponding to $50^{\circ}$ up $80^{\circ}$, in steps of $10^{\circ}$ are also displayed but the yields are lower. The dotted lines are extracted from the work of Berger and Seltzer (1970) for $(0-0.5)^{\circ}$ and $(0-5)^{\circ}$.

Distributions angulaires et en énergie du rayonnement de freinage par électron incident de $30 \mathrm{MeV}$. Le convertisseur cylindrique est formé de $\mathrm{W}$, l'épaisseur est de $24 \mathrm{~g} / \mathrm{cm}^{2}$. Le diamètre est égal à l'épaisseur. Les lignes pleines correspondent au calcul actuel. Quelques valeurs des angles d'émission sont marquées sur la figure. Les résultats qui correspondent à l'intervalle de $50^{\circ}$ jusqu'à $80^{\circ}$, en pas de $10^{\circ}$, sont aussi dessinés, mais les rendements sont plus petits. Les lignes discontinues sont extraites du travail de Berger et Seltzer (1970) pour $(0-0,5)^{\circ}$ et $(0-5)^{o}$.

facility will deliver about $50 \mathrm{MeV}$ electron beam. The results concerning the $50 \mathrm{MeV}$ electron beams onto $\mathrm{W}$ are displayed in Figure 3. It seems that this energy is in fact suitable for our purpose because the yields of the forward spectra are only $20-30 \%$ lower in the vicinity of $E_{\gamma} \approx 15 \mathrm{MeV}$ than those obtained at $60 \mathrm{MeV}$ incident energy. This decrease is not dramatic, and we avoid the production of intense gamma yields in the $50-60 \mathrm{MeV}$ region. The next step is the study of the radiate bremsstrahlung emission from the UCx target, when the $50 \mathrm{MeV}$ electron beams impinge directly on the target (without converter). The ratio between the number of $\mathrm{C}$ atoms versus that of $\mathrm{U}$ is 2.5 and the density of the mixture is $3.6 \mathrm{~g} / \mathrm{cm}^{3}$. Using the formulae presented in the previous section, the range of $50 \mathrm{MeV}$ electrons in the mixture was calculated as about $14.5 \mathrm{~g} / \mathrm{cm}^{2}$, being not too different than that in the $\mathrm{W}$ converter. Accordingly, for a comparison purpose with the results corresponding to the $\mathrm{W}$ converter, the thickness was taken also $30 \mathrm{~g} / \mathrm{cm}^{2}$ (approximately twice the range). The spectra obtained for the UCx target are presented in Figure 4. Practically, the forward direction spectra have the same 


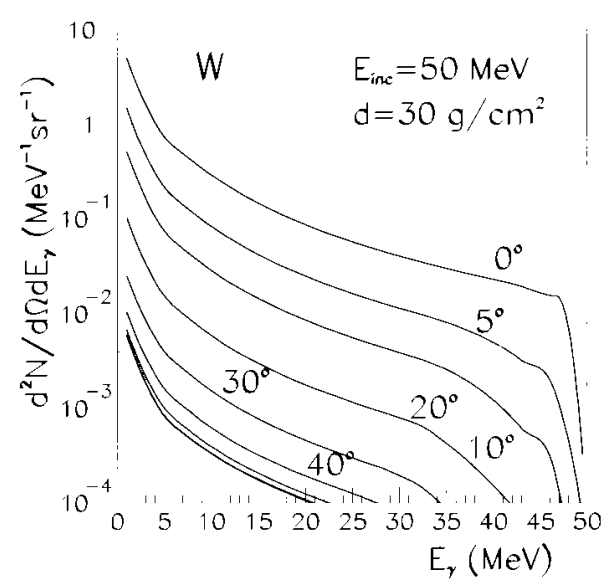

Figure 3 - Angular and energy distributions of the bremsstrahlung emission per 50 MeV incident electron. The cylindrical converter is made from $W$, the thickness is $30 \mathrm{~g} / \mathrm{cm}^{2}$. The diameter is equal to the thickness. The full lines correspond to the present calculations. Some emission angles are marked on the plot. The results corresponding to $50^{\circ}$ up $80^{\circ}$, in steps of $10^{\circ}$ are also displayed but the yields are lower.

Distributions angulaires et en énergie du rayonnement de freinage par électron incident de $50 \mathrm{MeV}$. Le convertisseur cylindrique est formé de W, l'épaisseur est de $30 \mathrm{~g} / \mathrm{cm}^{2}$. Le diamètre est égal à l'épaisseur. Les lignes pleines correspondent au calcul actuel. Quelques valeurs des angles d'émission sont marquées sur la figure. Les résultats qui correspondent à l'intervalle de $50^{\circ}$ jusqu'à $80^{\circ}$, en pas de $10^{\circ}$, sont aussi dessinés, mais les rendements sont plus petits.

shapes as those in the case of the $W$ converter. Having in mind that focusing the electron beam directly on the fissionable target, the bremsstrahlung radiation emitted in all the directions enhances the fission events number, it can be expected that the neutron rich nuclei productivity of the source increases. By placing the intermediate converter, a large amount of photons emitted at larger angles cannot reach the target.

The source term (emission rate) for $50 \mathrm{MeV}$ electrons impinging on $\mathrm{UCx}$ and $\mathrm{W}$ targets was calculated in order to estimate the shielding of the combination. The behavior of the source term is displayed in Figure 5 and compared with the NRCP $N^{\circ} 51$ (NRCP, 1977) where the energy is $100 \mathrm{MeV}$ for high Z targets. Our units are chosen in such a way to have a direct comparison with the published reference. Our values agree with the recommended ones, but indicates also that in the forward direction it appear that the radiation shielding must be reinforced. Our source term results are computed without taking into account the self-attenuation of photons in the target, so that are valid for all the target geometries. 


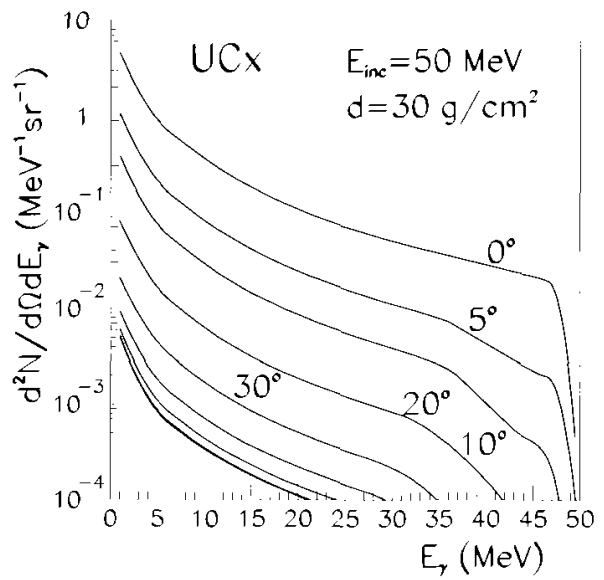

Figure 4-Angular and energy distributions of the bremsstrahlung emission per $50 \mathrm{MeV}$ incident electron. The cylindrical converter is made from UCx, the thickness is $30 \mathrm{~g} / \mathrm{cm}^{2}$. The diameter is equal to the thickness. The full lines correspond to the present calculations. Some emission angles are marked on the plot. The results corresponding to $50^{\circ}$ up $80^{\circ}$, in steps of $10^{\circ}$ are also displayed but the yields are lower.

Distributions angulaires et en énergie du rayonnement de freinage par électron incident de $50 \mathrm{MeV}$. Le convertisseur cylindrique est formé de UCx, l'épaisseur est de $30 \mathrm{~g} / \mathrm{cm}^{2}$. Le diamètre est égal à l'épaisseur. Les lignes pleines correspondent au calcul actuel. Quelques valeurs des angles d'émission sont marquées sur la figure. Les résultats qui correspondent à l'intervalle de $50^{\circ}$ jusqu'à $80^{\circ}$, en pas de $10^{\circ}$, sont aussi dessinés, mais les rendements sont plus petits.

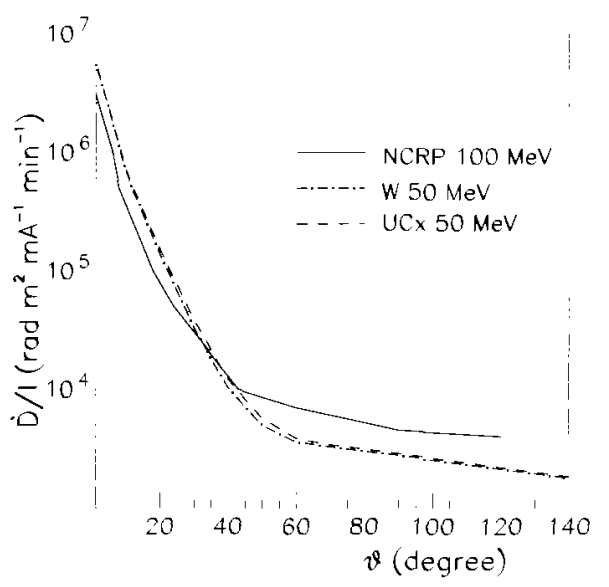

Figure 5 - Source term as function of the angle for the emission of photons from UCx and W targets compared with reference data. This quantity allows the determination of the dose for different intensities of the incident electron beam at different distances from the target.

Comparaison du terme source en fonction de l'angle d'émission des rayons gamma pour les cibles de UCx avec des donnés de référence. Cette grandeur permet de déterminer les doses pour différentes intensités du faisceau d'électrons incidents à certaines distances de la source. 


\section{Conclusion}

This study gives evidence for the advantages obtained by eliminating the intermediate converter and offers the possibility to estimate the required radiation shield of our combination. Furthermore, the numerical code developed will permit the evaluation of the efficiency dependence for different quantities of the neutron rich ion source (such as the number of fission events) in order to optimize the yields. Further studies are now being done in order to determine the fission production rate and the radiological issues (exposure to induced activity, fission gaseous products, ...).

Acknowledgements. This work was sponsored by the European Contracts SPIRAL II No. ERB 4062 PL 975009, No. FMGE CT 980100 and by the IDRANAP European Center of Excellence.

\section{REFERENCES}

Ahlen S.P. (1980) Theoretical and experimental aspects of the energy loss of relativistic heavily ionizing particles, Rev. Mod. Phys. 52, 121-173.

Berger M.J., Seltzer S.M. (1970) Bremsstrahlung and photoneutron from thick tungsten and tantalum targets, Phys. Rev. C 2, 621-631.

Clapier F., Mueller A.C., Obert C., Bajeat O., Ducourtieux M., Ferro A., Horbowa A., Kotfila L., Lau C., Lefort H., Kandri-Rody S., Pauwels N., Poitier J.C., Proust J., Putaux J.C., Liang C.F., Paris P.,Vilari A.C.C., Lichtenhaler R., Maunoury L., Lettry J. (1998) Exotic beams produced by fast neutrons, Phys. Rev. ST Accel. Beams 1, $013501(3)$.

Davisson C.M. (1968) Gamma-ray attenuation coefficients. In: Alpha-, beta- and gamma-ray spectroscopy (K. Sieghbahn, Ed.) appendix 1, pp. 827-843. North Holland Publ. Co. Amsterdam.

Diamond W.T. (1999) A radioactive ion beam facility using photofission, Nucl. Instrum. Meth. A 432 , 471-482.

Kandri-Rody S., Obert J., Cottereau E., Bajeat O., Ducourtieux M., Lau C., Lefort H., Potier J.C., Putaux J.C., Clapier F., Lettry J., Mueller A.C., Pauwels N., Proust J., Liang C.F., Paris P., Ravn H.L., Roussiere B., Sauvage J., Scarpaci J.A., Le Blanc F., Galu G., Lhenry I., Von Egidy T., Antoni R. (2000) Exotic nuclei produced by fast neutrons in a liquid uranium target, $\mathrm{Nucl}$. Instrum. Meth. B 160, 1-6.

Knop G., Paul W. (1968) Interaction of electrons and $\alpha$-particles with matter. In: Alpha-, beta- and gamma-ray spectroscopy (K. Sieghbahn, Ed.) Chap. 1, pp. 1-25. North Holland Publ. Co., Amsterdam.

Koch H.W., Motz J.W. (1950) Bremsstrahlung cross-section formulas and related data, Rev. Mod. Phys. 31, 920-955.

Leo W.R. (1987) Passage of radiation through matter. In Techniques for nuclear and particle physics experiments, Chap. 2, pp. 17-68. Springer Verlag, Berlin, Heildelberg, New York.

McKinley Jr W.A., Feshbach H. (1948) The Coulomb scattering of relativistic electrons by nuclei, Phys. Rev. 74(12), 1759-1763.

Mirea M., Bajeat O., Clapier F., Ibrahim F., Mueller A.C., Pauwels N., Proust J. (2001) Modeling a neutron rich nuclei source, Eur. Phys. J. A 11, 59-78. 


\section{MODELING THE BREMSSTRAHLUNG OF 30-60 MEV ELECTRONS}

Mirea M., Bajeat O., Clapier F., Essabaa S., Groza L., Ibrahim F., Kandri-Rody S., Mueller A.C., Pauwels N., Proust J. (2002) Exploratory analysis of a neutron-rich nucleii source based on photo-fission, Report IPNO 02-04.

NCRP (1977) publication $n^{\circ}$ 51, Radiation Protection Design Guidelines for 0.1-100 MeV Particle Accelerator Facilities, Washington, DC.

Pages L., Bertel E., Joffre H., Sklavenitis L. (1972) Energy loss, range, and bremsstrahlung yield for $10 \mathrm{keV}$ to $100 \mathrm{MeV}$ electrons in various elements and chemical compounds, At. Data 4, 1-127.

Scott W.T. (1963) The theory of small angle multiple scattering of fast charged particles, Rev. Mod. Phys. 35, 231-313.

Sternheimer R.M. (1952) The density effect for the ionization loss in various materials, Phys. Rev. 88, 851-859.

Sternheimer R.M. (1961) Interaction of radiation with matter. In: Methods of experimental physics (L.C.L. Yuan, C.S. Wu, Eds.) Vol. 5, Part A, Chap. 1.1, pp. 1-89. Academic Press, New York.

Tabata. T, Ito R. (1979) Approximation to Landau's distribution functions for the ionization energy loss of fast particles, Nucl. Instrum. Meth. 158, 521-523.

Tsai Y.S., Whitis V. (1966) Thick-target bremsstrahlung and target considerations for secondaryparticle production by electrons, Phys. Rev. 149, 1248-1256. 